\title{
Recomendaciones para la definición de la política de vacunación contra el virus del papiloma en México
}

\author{
Comité Asesor Externo para la Definición de la Política de Vacunación \\ contra el Virus del Papiloma en México*
}

$E^{1}$ cáncer cervical es tan sólo un reflejo de la enorme _iniquidad social que sobrellevan las mujeres que lo padecen. ${ }^{1}$ En efecto, en los países con elevada incidencia y mortalidad por tumores prevenibles se han observado grandes disparidades en cuanto al acceso a la atención y tratamiento oportunos, lo que en gran medida refleja las enormes desigualdades entre sus ciudadanos. ${ }^{2}$ En lo que refiere a la prevención y control del cáncer cervical, se han identificado diversas dimensiones de iniquidad, la primera de ellas es el acceso desigual a los servicios de salud en las mujeres pobres. ${ }^{3}$ Adicionalmente, se ha documentado una mala calidad y escasa infraestructura en programas de prevención y control del cáncer cervical en áreas rurales. ${ }^{4}$ En el momento actual, la prevención primaria con vacunación contra el virus de papiloma humano (VPH) es inaccesible debido al elevado costo, ${ }^{5,6}$ y finalmente, existe desigualdad de género, ${ }^{7}$ no sólo porque la enfermedad es propia de las mujeres, sino porque en muchas poblaciones se privilegia la salud de los hombres en detrimento de la de las mujeres. ${ }^{8}$

Durante los últimos 25 años se han presentado oficialmente en México más de 100000 muertes por cáncer cervical, y a partir de 2006 se constituyó en la segunda causa de muerte por tumores malignos en la mujer, después del cáncer de mama. A pesar de que existe una disminución significativa de la mortalidad por cáncer cervical a partir de la década de los noventa, en gran medida atribuible a la disminución de las tasas de natalidad, ${ }^{9}$ persiste en México un elevado incremento en el número de casos por cáncer cervical en áreas rurales, a lo que hay que dar una respuesta de prevención y control inmediata. Por este motivo deben implementarse acciones inmediatas para enfrentar esta agenda inconclusa y dar una respuesta innovadora a las miles de mujeres mexicanas que actualmente sufren neoplasia cervical.

\section{Consideraciones generales de las vacunas contra VPH}

Los tipos de VPH 16 y 18 son responsables de alrededor del $70 \%$ de los casos de cáncer cervical invasor, ${ }^{10}$ así como de una alta proporción de cáncer anogenital ${ }^{11}$ y un menor porcentaje de neoplasias en cuello y cabeza. ${ }^{12}$

\footnotetext{
* El Comité está integrado por Eduardo Lazcano-Ponce, ${ }^{(1)}$ Jorge Salmerón-Castro, ${ }^{(2)}$ Alejandro García-Carrancá, ${ }^{(3,4)}$ Carlos Aranda-Flores, ${ }^{(5)}$ Vicente MadridMarina, ${ }^{(1)}$ César Misael Gómez-Altamirano( ${ }^{(6)}$ y Olga Georgina Martínez-Montañez. ${ }^{(7)}$

(I) Instituto Nacional de Salud Pública. México.

(2) Instituto Mexicano del Seguro Social. México.

(3) Instituto Nacional de Cancerología de México.

(4) Instituto de Investigaciones Biomédicas de la Universidad Nacional Autónoma de México.

(5) Instituto Nacional de Perinatología. México.

(6) Centro Nacional para la Salud de la Infancia y la Adolescencia. México.

(7) Centro Nacional de Equidad de Género y Salud Reproductiva. México.
} 
Recientemente, en varios países de ingresos altos y en algunos de ingresos medios, se introdujeron en los programas de inmunización dos vacunas para la profilaxis de las infecciones por VPH con los tipos 16 y 18:13

- Cervarix. Vacuna bivalente, con dos proteínas antigénicas no infecciosas, para VPH 16 y 18.

- Gardasil. Vacuna tetravalente, con cuatro proteínas antigénicas para VPH 16, 18, 6 y 11, las dos últimas para la prevención de verrugas genitales y/o papilomatosis respiratoria recurrente.

\section{Resultados de los ensayos de vacunas profilácticas contra VPH}

Existen diversos hallazgos claves respecto a la efectividad de las vacunas profilácticas contra $\mathrm{VPH}_{1}, 14,15,16$ entre ellos se encuentran:

1. Eficacia del $100 \%$ en la prevención de lesiones relacionadas con VPH 16-18, en mujeres antes del inicio de su vida sexual o que estén libres de la infección.

2. En mujeres con infección prevalente al VPH 16/18, la aplicación de vacunas no ha evidenciado un efecto. $^{17}$

3. Buena persistencia de anticuerpos durante 7 años. ${ }^{18}$

4. Aceptable perfil de seguridad. ${ }^{19}$

A este respecto, los resultados de los ensayos clínicos que se han desarrollado en más de 40 áreas geográficas en todo el mundo, ${ }^{20}$ han referido que las vacunas contra $\mathrm{VPH}$ son altamente eficaces contra infección persistente por VPH a los 6 y 12 meses $^{21}$ y contra cualquier tipo de neoplasia intraepitelial cervical; ${ }^{22}$ en vacunas tetravalentes, también han sido eficientes contra lesiones genitales externas. ${ }^{23}$ Actualmente, sin embargo, existen diversas controversias que serán dilucidadas en el futuro. Una de ellas se refiere a la vacuna bivalente contra VPH 16 y 18. Sus patrocinadores han referido un mayor nivel de eficacia cuando se utiliza adyuvante AS04, según lo que revela un estudio de comparación. ${ }^{24}$ Asimismo, también se ha sugerido un mayor nivel de inmunogenicidad, ${ }^{25}$ cuyo impacto, en comparación con la vacuna tetravalente, no es conocido y posiblemente no se conocerá en el mediano plazo.

En relación con la vacuna tetravalente contra los VPH 6, 11, 16 y 18, se ha documentado la utilidad para prevenir otro tipo de lesiones, entre ellas cánceres anogenitales, que incluyen el vulvar, vaginal y cáncer anogenital en hombres que tienen sexo con hombres. ${ }^{26}$ Asimismo, se ha documentado la prevención de otras enfermedades, como la papilomatosis respiratoria recurrente, ${ }^{27}$ las verrugas genitales, y una pequeña fracción prevenible de cáncer orofaríngeo. ${ }^{28}$

Actualmente, se han iniciado líneas de investigación de VPH asociado a cáncer de piel $^{29} \mathrm{y}$ existen nuevos desarrollos tecnológicos con nuevas generaciones de vacunas polivalentes contra VPH, que incluirán una protección contra 9 genotipos. ${ }^{30}$

Una limitación del uso de vacunas contra VPH es que éstas no tienen impacto cuando se administran en sujetos que cursan con infecciones de los tipos de VPH contenidos en la vacuna. Recientemente, sin embargo, se ha reportado que mujeres con seropositividad a VPH 16 y 18, en ausencia de infección clínica por VPH (ADN de VPH negativo), pueden beneficiarse con el uso de la vacuna. $^{31}$

\section{Inmunogenicidad de las vacunas contra VPH}

Con respecto a la inmunogenicidad de las vacunas, no se ha identificado una correlación de protección inmunológica en los vacunados, es decir, no se conoce el nivel mínimo requerido de anticuerpos para proteger contra la infección. Lo que se ha establecido es la correlación entre presencia de anticuerpos y efecto profiláctico. Sin embargo, la inmunogenicidad se ha relacionado con la edad, y los niveles de anti-VPH en el mes séptimo con un esquema de tres dosis son estadísticamente más altos en las adolescentes menores de 12 años, en comparación con las adolescentes y mujeres de más edad. ${ }^{32}$

En los estudios de inmunogenicidad llevados a cabo en los ensayos clínicos de vacunas contra VPH, es consistente la observación de un mayor pico de anticuerpos al séptimo mes del inicio de la vacunación. ${ }^{33}$ Posteriormente, se produjo un descenso paulatino de las GMT (títulos medios geométricos de $\mathrm{IgG}$ ) y al llegar al mes 24 se estabilizaron, manteniéndose constantes hasta el mes $60 .^{34}$

La existencia de una respuesta inmunitaria de memoria se puso en evidencia en los sujetos previamente seropositivos frente a un VPH 16/18, cuando se les administró la vacuna y se observó un aumento considerable del título de anticuerpos frente a dicho VPH. ${ }^{35}$ Por otra parte, y más importante aún, es el hecho de que a un subconjunto de individuos (aproximadamente 80 sujetos), se les administró una dosis extra (cuarta dosis) de vacuna a los 60 meses de la primera vacunación y se detectó una rápida y vigorosa respuesta inmunitaria de memoria frente a los cuatro tipos de VPH incluidos en la vacuna, que excedió los niveles de GMT observados al séptimo mes de iniciada la vacunación. ${ }^{36}$

Para la vacuna bivalente, la eficacia en mujeres se estableció en dos ensayos clínicos realizados en niñas y 
adolescentes de 10 a 17 años de edad. En todos los casos hubo seroconversión para ambos tipos de VPH, 16 y 18, después de la tercera dosis (en el mes 7), con unos GMT al menos 2 veces más elevados en comparación con mujeres vacunadas de 15 a 25 años. ${ }^{37}$

Se conoce que la respuesta inducida de anticuerpos por la vacuna es mucho más alta que la que ocurre con la infección natural y que la respuesta inmune de las niñas de 9 a 11 años después de dos dosis de vacuna es similar o mayor a la obtenida después de tres dosis en mujeres de 16 a 26 años de edad, para las cuales se ha probado la eficacia de la vacuna. ${ }^{38}$

De hecho, la respuesta inmune que genera la vacuna puede ser hasta 100 veces mayor a la inducida por la inmunidad natural. Con estos antecedentes, y desde la perspectiva de los servicios de salud, un esquema inicial con dos dosis y una tercera extendida tiene ventajas en su administración, ya que la organización de las estrategias para completar un esquema inicial de dos dosis, con una tercera a los 60 meses, es más sencilla.

\section{Antecedentes de vacunación con periodicidad de las dosis de 0, 6 y 60 meses}

El National Advisory Committee on Inmunization (NACI) en Canadá ha identificado la inmunogenicidad de dos dosis de vacuna y actualmente desarrolla una intervención en tres provincias de Canadá: British Columbia, Quebec y Nova Scotia que plantea el esquema con vacunación a los 0, 6 y 60 meses. ${ }^{39}$ Esta iniciativa es una de las 10 más relevantes que se implementan sobre la vacuna contra VPH en Canadá. Los argumentos para este tipo de intervención son los siguientes:

- La inducción de anticuerpos por la vacuna es más alta que la producida por la exposición natural al virus.

- La respuesta inmune de las niñas de 9 a 11 años de edad es similar a la obtenida después de tres dosis en mujeres entre los 16 y los 26 años de edad.

- De acuerdo con estudios puente, nada indica que la vacuna será menos efectiva si se administra bajo un esquema extendido. Las intervenciones que se realizan regionalmente en Canadá son para determinar la inmunogenicidad y eficacia de este esquema.

- La administración de la tercera dosis será en el momento en el que se requiere la máxima protección, es decir, alrededor del inicio de las relaciones sexuales.

- Una dosis administrada a los cinco años de la primera vacunación tuvo una respuesta de anticuerpos más alta que la obtenida durante la vacunación primaria. Esta respuesta también ha sido observada con la vacuna contra la hepatitis $B$.

\section{Conclusiones y recomendaciones del Strategic Advisory Group of Experts (SAGE)}

Las siguientes son las conclusiones y recomendaciones del Strategic Advisory Group of Experts (SAGE) sobre inmunizaciones, de la Organización Mundial de la Salud, en la reunión de noviembre de 2008: ${ }^{40}$

- La introducción de la vacuna profiláctica contra el virus del papiloma humano (VPH) puede ser de gran beneficio en el ámbito mundial.

- Debido a la magnitud del cáncer cervical y otras enfermedades relacionadas a la infección con VPH, se recomienda que la vacunación contra el VPH se incluya en los programas nacionales de inmunización, considerando que la prevención del cáncer cervical constituye un problema de salud pública prioritario.

- La introducción de las vacunas debe ser factible, sostenible y su financiamiento debe estar asegurado, por lo que ha de considerarse el costoefectividad de las estrategias.

- La vacunación es más eficaz en las mujeres que no han estado expuestas al VPH, por lo que el grupo blanco primario pueden ser las niñas y adolescentes entre los 9 y los 13 años de edad. Las decisiones finales deben basarse en los datos de edad de inicio de las relaciones sexuales y la factibilidad de facilitar la vacuna en escuelas, servicios de salud y la comunidad.

- Es recomendable la vacunación de adolescentes de mayor edad como segundo grupo prioritario para la vacunación, en el caso de que sea económicamente factible, sostenible y costo-efectivo, y que además no sea causa de menor inversión de recursos para vacunar al primer grupo prioritario y que la proporción de adolescentes de mayor edad no expuestas al VPH sea significativa.

- Actualmente no se recomienda vacunar a los varones.

- Los estudios piloto en varios países sugieren que las estrategias basadas en la administración de la vacuna en las escuelas es factible.

- Deben utilizarse estrategias que sean compatibles con la infraestructura de abasto, la capacidad de la red fría, y que además sean costo-efectivas y sostenibles para alcanzar la más alta cobertura posible.

- Si la introducción se realiza por fases, deben privilegiarse las adolescentes con menor probabilidad 
de tener acceso al tamizaje de cáncer cervical en la vida adulta.

- La vacunación debe introducirse como parte de estrategias coordinadas de prevención del cáncer cervical y otras enfermedades relacionadas a la infección con VPH, como la educación para reducir las conductas que incrementan el riesgo de infectarse, la detección del cáncer cervical, así como el diagnóstico y tratamiento de las lesiones precursoras y del cáncer. Sin embargo, la vacunación no debe retrasarse en países donde una o más de esas intervenciones no pueda ser implementada al tiempo de la introducción de la vacuna.

- Las recomendaciones para el tamizaje del cáncer cervical deben seguir siendo las mismas en las mujeres vacunadas.

Después de introducir la vacunación se deberá:

- Medir la cobertura por edad y por municipio, al tiempo que los registros deberán mantenerse por periodos prolongados.

- Vigilar los efectos indeseados y la seguridad de la vacuna.

- Considerar el establecimiento de vigilancia centinela para el monitoreo del impacto de la vacunación sobre la prevalencia de la infección por $\mathrm{VPH}$, la incidencia de anormalidades cervicales, lesiones precursoras, condilomas acuminados, así como la incidencia y mortalidad por cáncer cervical.

\section{Ventajas de la utilización en México de un esquema a los meses 0,6 y 60}

- Es más sencillo organizar una estrategia de vacunación inicial con las dosis a los 0 y 6 meses, en niñas de 9 años de edad (cuarto año de primaria). Estas niñas pueden ser vacunadas durante las semanas de vacunación en las que se visitan escuelas para aplicar dosis de refuerzo a niñas en sexto año de primaria.

- Se amplía la cobertura de vacunación en un tercio, ya que con los mismos recursos se vacunarán más niñas inicialmente. Cuando sea necesario aplicar la tercera dosis, la vacuna tendrá un costo más accesible, por lo que en términos económicos será más costo-efectiva y sostenible.

- La tercera dosis se aplicaría a los 14 años (tercero de secundaria), edad previa al inicio de relaciones sexuales en la mayoría de las adolescentes. El alza en los anticuerpos con la tercera dosis puede asegurar una mayor protección contra la infección persistente y sus consecuencias.

- La aplicación de la tercera dosis en el tercer año de secundaria, puede ser combinada más fácilmente con educación sexual para evitar conductas de mayor riesgo para adquirir la infección con VPH y con información sobre la detección del cáncer cervical en la vida adulta y en general con educación sobre salud reproductiva.

- El monitoreo de los títulos de anticuerpos en las cohortes de adolescentes mostrará si es necesario adelantar la tercera dosis, sin riesgo de exponerlas a la infección, con la seguridad de que una proporción significativa de adolescentes no habrá iniciado actividad sexual a los 14 años.

- Aun cuando se iniciara vida sexual antes de la tercera dosis, existiría una potencial protección en el periodo de riesgo de infección, que existe durante la actividad sexual.

- Existe el antecedente de la vacuna contra hepatitis $\mathrm{B}$, que originalmente tenía consideradas 3 dosis, mientras que actualmente se recomienda un esquema de vacunación de 2 dosis.

\section{Vacunación contra VPH en México: el reto de países con ingresos medios}

Las vacunas contra VPH se encuentran disponibles comercialmente, pero ningún país en Latinoamérica las ha introducido en el esquema de vacunación universal, a pesar de que existe una fuerte presión de la industria farmacéutica en países con ingresos medios para incorporarla como una política de salud. La región de Latinoamérica tiene una incidencia y mortalidad por cáncer cervical entre 3 y 10 veces mayor que países industrializados ${ }^{41}$ y el PIB es un criterio recomendado por la OMS para implementar políticas de inmunización contra VPH; consecuentemente el PIB y el presupuesto en salud son considerablemente más bajos en Latinoamérica y no hay una justificación para implementar programas de vacunación poblacional contra VPH, ante el elevado costo. Un análisis de costo-efectividad de vacuna contra VPH en México determinó que las estrategias de prevención secundaria son más costo-efectivas para mujeres adultas, con la utilización del Papanicolaou y captura de híbridos. Para que la vacunación contra VPH sea costo-efectiva, deberá tener un costo no mayor de 18 USD por dosis.

Actualmente existen políticas de instrumentación de nuevas estrategias de prevención y control del cáncer cervical en México, con alternativas de prevención acordes al grupo de edad, que se han implementado a 
iniciativa del gobierno federal en áreas marginadas. Estos abordajes son creativos, porque son un intento por integrar en los modelos de prevención del cáncer cervical, a las madres (mujeres adultas) y a las hijas, de acuerdo con su nivel de prevención primario o secundario.

\section{Consideración ética en salud pública}

La adopción de un esquema extendido de vacunación contra el VPH se justifica porque es seguro, se alcanzan niveles de inmunogenicidad adecuados, la logística de aplicación es más práctica y se incrementa la cobertura de detección en un tercio. Es una obligación del Estado combatir las desigualdades de salud y brindar protección en el ámbito de la prevención primaria y secundaria, con la mejor tecnología disponible en una elevada relación costo-beneficio. El esquema extendido atiende esta responsabilidad, ya que al aumentar la cobertura de protección en las niñas, se maximiza el beneficio ante recursos limitados, a diferencia de la otra propuesta en la que se salvan vidas pero sólo se beneficia a unas cuantas personas.

\section{Recomendación del grupo asesor de vacunación contra VPH}

- Se recomienda un esquema de vacunación contra VPH a los 0, 6 y 60 meses en niñas de 9 años.

- Este esquema incrementará la cobertura de prevención primaria en niñas a los 9 años.

- El esquema extendido a los 0, 6 y 60 meses no se recomienda en adolescentes mayores de 12 años.

- La vacunación debe integrarse al fortalecimiento de las acciones de prevención y control del cáncer cervicouterino a través de la detección y el tratamiento oportunos y de calidad.

- Se debe incorporar un sistema de vigilancia epidemiológica a través de un laboratorio central que cuantifique niveles de anticuerpos contra VPH 16 y 18 en la población intervenida, que permita evaluar adicionalmente la respuesta inmune con dos dosis y estimar el posible efecto de protección.

- La tercera dosis tendrá menor costo, al diferirla 60 meses.

- Los ahorros generados deberán fortalecer la detección secundaria.

Finalmente, podemos afirmar que la implementación de programas integrales e innovadores de prevención de cáncer cervical es factible, pero para hacer eficiente dicha intervención deben implementarse asimismo mecanismos de control de calidad en cada uno de los elementos del proceso.

\section{Referencias}

I. Newmann SJ, Garner EO. Social inequities along the cervical cancer continuum: a structured review. Cancer Causes Control 2005; I6(I):63-70. 2. Chu KC, Miller BA, Springfield SA. Measures of racial/ethnic health disparities in cancer mortality rates and the influence of socioeconomic status. J Natl Med Assoc 2007;99(10): 1092-I I00, I I02-I 104.

3. Ward E, Jemal A, Cokkinides V, Singh GK, Cardinez C, Ghafoor A, et al. Cancer disparities by race/ethnicity and socioeconomic status. CA Cancer J Clin 2004;54(2):78-93.

4. Palacio-Mejía LS, Rangel-Gómez G, Hernández-Avila M, Lazcano-Ponce E. Cervical cancer, a disease of poverty: mortality differences between urban and rural areas in Mexico. Salud Publica Mex 2003;45 Suppl 3:S3I5-25. 5.Tsu VD, Levin CE. Making the case for cervical cancer prevention: what about equity? Reprod Health Matters 2008; 16(32): 104-I I2.

6. Goldie SJ, O'Shea M, Diaz M, Kim SY. Benefits, cost requirements and cost-effectiveness of the HPVI6, I8 vaccine for cervical cancer prevention in developing countries: policy implications. Reprod Health Matters 2008; 16(32):86-96.

7. Loxton D, Powers J, Schofield M, Hussain R, Hosking S. Inadequate cervical cancer screening among mid-aged Australian women who have experienced partner violence. Prev Med 2008 Nov 5. [Epub ahead of print] 8. Gerend MA, Barley J. Human Papillomavirus Vaccine Acceptability Among Young Adult Men. Sex Transm Dis 2008 Sep 30. [Epub ahead of print]

9. Lazcano-Ponce E, Palacio-Mejia LS,Allen-Leigh B,Yunes-Diaz E,Alonso P, Schiavon R, et al. Decreasing cervical cancer mortality in Mexico: effect of Papanicolaou coverage, birthrate, and the importance of diagnostic validity of cytology. Cancer Epidemiol Biomarkers Prev 2008; I7(I0):2808-28I7.

10. Muñoz N, Bosch FX, de Sanjosé S, Herrero R, Castellsagué X, Shah KV, et al. International Agency for Research on Cancer Multicenter Cervical Cancer Study Group. Epidemiologic classification of human papillomavirus types associated with cervical cancer. N Engl J Med 2003;348(6):5 I8-527.

II. Paavonen J. Human papillomavirus infection and the development of cervical cancer and related genital neoplasias. Int J Infect Dis 2007; I I Suppl 2:S3-9.

12. Campisi G, Panzarella V, Giuliani M, Lajolo C, Di Fede O, Falaschini $\mathrm{S}$, et al. Human papillomavirus: its identity and controversial role in oral oncogenesis, premalignant and malignant lesions (review). Int J Oncol 2007;30(4):8I3-823.

13. McIntyre PB, Brotherton JM, Burgess MA, Kemp AS. More data from Australia on sensitivity to HPV vaccine. BMJ 2009;338:b26.

14. Perez G, Lazcano-Ponce E, Hernandez-Avila M, García PJ, Muñoz N, Villa $L L$, et al. Safety, immunogenicity, and efficacy of quadrivalent human papillomavirus (types 6, II, I6, I8) LI virus-like-particle vaccine in Latin American women. Int J Cancer 2008; |22(6): |3|I-13|8.

15. Joura EA, Leodolter S, Hernandez-Avila M,Wheeler CM, Perez $G$, Koutsky LA, et al. Efficacy of a quadrivalent prophylactic human papillomavirus (types 6, II, I6, and I8) LI virus-like-particle vaccine against high-grade vulval and vaginal lesions: a combined analysis of three randomised clinical trials. Lancet 2007;369(9574):1693-1702.

16. Paavonen J, Jenkins D, Bosch FX, Naud P, Salmerón J, Wheeler CM, et al. Efficacy of a prophylactic adjuvanted bivalent LI virus-like-particle vaccine against infection with human papillomavirus types 16 and 18 in young women: an interim analysis of a phase III double-blind, randomised controlled trial. Lancet 2007;369(9580):2161-2I70.

17. Hildesheim A, Herrero R, Wacholder S, Rodriguez AC, Solomon D, Bratti MC, et al. Costa Rican HPVVaccine Trial Group. Effect of human papillomavirus $16 / 18 \mathrm{LI}$ viruslike particle vaccine among young women with preexisting infection: a randomized trial. JAMA 2007;298(7):743-753. 18. Giuliano AR, Lazcano-Ponce E, Villa L, Nolan T, Marchant C, Radley D, et al. Impact of baseline covariates on the immunogenicity of a quadrivalent 
(types 6, II, 16, and 18) human papillomavirus virus-like-particle vaccine. J Infect Dis 2007; 196(8): II53-II62.

19. Reisinger KS, Block SL, Lazcano-Ponce E, Samakoses R, Esser MT, Erick J, et al. Safety and persistent immunogenicity of a quadrivalent human papillomavirus types $6,1 \mathrm{I}, 16,18 \mathrm{LI}$ virus-like particle vaccine in preadolescents and adolescents: a randomized controlled trial. Pediatr Infect Dis J 2007;26(3):20I-209.

20. Schiller JT, Castellsagué X,Villa LL, Hildesheim A.An update of prophylactic human papillomavirus $L I$ virus-like particle vaccine clinical trial results. Vaccine 2008;26 Suppl 10:K53-6I.

21. Kovács K,Varnai AD, Bollmann M, Bankfalvi A, Szendy M, Speich N, et al. Prevalence and genotype distribution of multiple human papillomavirus infection in the uterine cervix: a 7.5-year longitudinal study in a routine cytology-based screening population in West Germany.J Med Virol 2008;80(10):1814-1823.

22. Sigurdsson K, Sigvaldason H, Gudmundsdottir T, Sigurdsson R, Briem $H$. The efficacy of HPV I6//8 vaccines on sexually active 18-23 year old women and the impact of HPV vaccination on organized cervical cancer screening. Acta Obstet Gynecol Scand 2008;22: I-9.

23. Rambout L, Hopkins L, Hutton B, Fergusson D. Prophylactic vaccination against human papillomavirus infection and disease in women: a systematic review of randomized controlled trials. CMAJ 2007; I77(5):469-479.

24. Dessy FJ, Giannini SL, Pinto LA, Wettendorff MA. Correlation between direct ELISA, single epitope-based inhibition ELISA and pseudovirionbased neutralization assay for measuring anti-HPV-I6 and anti-HPV- 18 antibody response after vaccination with the ASO4-adjuvanted HPV-16/18 cervical cancer vaccine. Hum Vaccin 2008;4(6):425-434.

25. Petäjä T, Keränen H, Karppa T, Kawa A, Lantela S, Siitari-Mattila M, et al. Immunogenicity and safety of human papillomavirus (HPV)-16/18 ASO4adjuvanted vaccine in healthy boys aged $10-18$ years. J Adolesc Health 2009;44(I):33-40

26. Gillison ML, Chaturvedi AK, Lowy DR. HPV prophylactic vaccines and the potential prevention of noncervical cancers in both men and women. Cancer 2008; I I3(I0 Suppl):3036-3046.

27. Chesson HW, Forhan SE, Gottlieb SL, Markowitz LE. The potential health and economic benefits of preventing recurrent respiratory papillomatosis through quadrivalent human papillomavirus vaccination. Vaccine 2008;26(35):45।3-45 I8.

28. Andrews E, Seaman WT,Webster-Cyriaque J. Oropharyngeal carcinoma in non-smokers and non-drinkers: A role for HPV. Oral Oncol 2008; I : 20

29. Feltkamp MC, de Koning MN, Bavinck JN, Ter Schegget J.

Betapapillomaviruses: Innocent bystanders or causes of skin cancer.J Clin Virol 2008;43(4):353-360.

30. Tovar JM, Bazaldua OV. New quadrivalent HPV vaccine developments. Postgrad Med 2008;120(4):14-16.
31. Paavonen J, Future II Study Group. Baseline demographic characteristics of subjects enrolled in international quadrivalent HPV (types 6/I ///6//8) vaccine clinical trials. Curr Med Res Opin 2008;24(6):1623-1634.

32. Giuliano AR, Lazcano-Ponce E,Villa L, Nolan T, Marchant C, Radley D, et al. Impact of baseline covariates on the immunogenicity of a quadrivalent (types 6, II, I6, and I8) human papillomavirus virus-like-particle vaccine.J Infect Dis 2007; 196(8): I I53-I I62. Epub 2007 Sep 17.

33. Reisinger KS, Block SL, Lazcano-Ponce E, Samakoses R, Esser MT, Erick J, et al. Safety and persistent immunogenicity of a quadrivalent human papillomavirus types $6,1 \mathrm{I}, 16,18 \mathrm{LI}$ virus-like particle vaccine in preadolescents and adolescents: a randomized controlled trial. Pediatr Infect Dis J 2007;26(3):20I-209.

34. Joura EA, Kjaer SK, Wheeler CM, Sigurdsson K, Iversen OE, Hernandez-Avila M, et al. HPV antibody levels and clinical efficacy following administration of a prophylactic quadrivalent HPV vaccine. Vaccine 2008;26(52):6844-6845I. Epub 2008 Oct I6.

35. Villa LL,Ault KA, Giuliano AR, Costa RL, Petta CA,Andrade RP, et al. Immunologic responses following administration of a vaccine targeting human papillomavirus Types 6, I I, I6, and I8.Vaccine 2006;24(2728):557।-5583.

36. Olsson SE, Villa LL, Costa RL, Petta CA, Andrade RP, Malm C, et al. Induction of immune memory following administration of a prophylactic quadrivalent human papillomavirus (HPV) types 6/I I/I6/18 LI virus-like particle (VLP) vaccine. Vaccine 2007;25(26):4931-4939.

37. Schwarz TF, Leo O. Immune response to human papillomavirus after prophylactic vaccination with AS04-adjuvanted HPV-16/18 vaccine: improving upon nature. Gynecol Oncol 2008; I I0(3 Suppl I):SI-I0. 38. Block SL, Nolan T, Sattler C, Barr E, Giacoletti KE, Marchant CD, et al. Comparison of the immunogenicity and reactogenicity of a prophylactic quadrivalent human papillomavirus (types 6, II, I6, and I8) LI virus-like particle vaccine in male and female adolescents and young adult women. Pediatrics 2006; I |8(5):2135-2/45.

39. Agence de la santé et des services sociaux de Montréal. Québec: HVP Vaccination. Questions and Answers for Practicioners. [Consulted on May 2009] Available at: http://www.santepub-mtl.qc.ca/Mi/vaccination/pdf/ VPHenglish2008.pdf.

40. Meeting of the immunization Strategic Advisory Group of Experts, November 2008-conclusions and recomendations. Wkly Epidemiol Rec 2009;84(I-2):9-12.

4I. Parkin DM,Almonte M, Bruni L, Clifford G, Curado MP, Piñeros M. Burden and trends of type-specific human papillomavirus infections and related diseases in the Latin America and Caribbean region.Vaccine 2008;26 Suppl I I:LI-I5. 